\title{
Dissecting Content and Context in Argumentative Relation Analysis
}

\author{
Juri Opitz and Anette Frank \\ Research Training Group AIPHES, \\ Leibniz ScienceCampus "Empirical Linguistics and Computational Language Modeling" \\ Department for Computational Linguistics \\ 69120 Heidelberg \\ \{opitz, frank\}@cl.uni-heidelberg.de
}

\begin{abstract}
When assessing relations between argumentative units (e.g., support or attack), computational systems often exploit disclosing indicators or markers that are not part of elementary argumentative units (EAUs) themselves, but are gained from their context (position in paragraph, preceding tokens, etc.). We show that this dependency is much stronger than previously assumed. In fact, we show that by completely masking the EAU text spans and only feeding information from their context, a competitive system may function even better. We argue that an argument analysis system that relies more on discourse context than the argument's content is unsafe, since it can easily be tricked. To alleviate this issue, we separate argumentative units from their context such that the system is forced to model and rely on an EAU's content. We show that the resulting classification system is more robust, and argue that such models are better suited for predicting argumentative relations across documents.
\end{abstract}

\section{Introduction}

In recent years we have witnessed a great surge in activity in the area of computational argument analysis (e.g. Peldszus and Stede (2013); Stab and Gurevych (2014b); Rasooli and Tetreault (2015); Stab et al. (2018)), and the emergence of dedicated venues such as the ACL Argument Mining workshop series starting in 2014 (Green et al., 2014).

Argumentative relation classification is a subtask of argument analysis that aims to determine relations between argumentative units $\mathrm{A}$ and $\mathrm{B}$, for example, A supports B; A attacks B. Consider the following argumentative units (1) and (2), given the topic (0) "Marijuana should be legalized":

(1) Legalizing marijuana can increase use by teens, with harmful results.

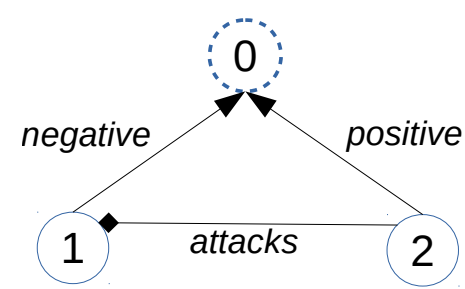

Figure 1: A graph representation of a topic (node w/ dashed line), two argumentative premise units (nodes w/ solid line), premise-topic relations (positive or negative) and premise-premise relations (here: attacks).

\section{(2) Legalization allows the government to set age restrictions on buyers.}

This example is modeled in Figure 1. It is clear that (1) has a negative stance towards the topic and (2) has a positive stance towards the topic. Moreover, we can say that (2) attacks (1). In discourse, such a relation is often made explicit through discourse markers: (1). However, (2); On the one hand (1), on the other (2); (1), although (2); Admittedly, (2); etc. In the absence of such markers we must determine this relation by assessing the semantics of the individual argumentative units, including (often implicit) world knowledge about how they are related to each other. ${ }^{1}$

In this work, we show that argumentative relation classifiers - when provided with textual context surrounding an argumentative unit's span are very prone to neglect the actual textual content of the EAU span. Instead they heavily rely on contextual markers, such as conjunctions or adverbials, as a basis for prediction. We argue that a system's capacity of predicting the correct relation based on the argumentative units' content is important in many circumstances, e.g., when an argumentative debate crosses document boundaries.

\footnotetext{
${ }^{1}$ In case of (1) and (2): By setting age restrictions on legalization of Marijuana, increased use by teens can be (expected to be) prevented, thus we can infer that (2) attacks (1).
} 
For example, the prohibition of marijuana debate extends across populations and countries - argumentative units for this debate can be recovered from thousands of documents scattered across the world wide web. As a consequence, argumentative relation classification systems should not be (immensely) dependent on contextual clues - in the discussed cross-document setting these clues may even be misleading for such a system, since source and target arguments can be embedded in different textual contexts (e.g., when (1) and (2) stem from different documents it is easy to imagine a textual context where (2) is not introduced by however but instead by an 'inverse' form such as e.g. moreover).

Contributions In Section $\S 3$ we describe argumentative relation classification systems and their features. Then, to assess the systems' dependency on context, we propose a three-way feature grouping: (i) features which access only the EAU span; (ii) features which access only the context of an EAU; (iii) features which access both EAU span and its context. Our experimental results ( $\S 4$ ) indicate that systems, when given the option, tend to focus on the context of an EAU, while neglecting its content. On the one hand, this leads to strong performance when EAUs appear sequentially in a rhetorically well structured argumentative monologue. Yet, on the other hand, we show that such systems can easily be fooled, e.g., when EAUs are extracted from different documents.

\section{Related Work}

It is well-known that the rhetorical and argumentative structure of texts bear great similarities. For example, Azar (1999); Green (2010); Peldszus and Stede (2013) observe that elementary discourse units (EDUs) in RST (Mann and Thompson, 1987) share great similarity with elementary argumentative units (EAUs) in argumentation analysis. ${ }^{2}$ Wachsmuth et al. (2018) experiment with a modified version of the Microtext corpus (Peldszus and Stede, 2016), which is an extensively annotated albeit small corpus. Similar to us, they separate argumentative units from discursive contextual markers. While Wachsmuth et al. (2018) conduct a human evaluation to investigate the separation of Logos and Pathos aspects of arguments,

\footnotetext{
${ }^{2}$ Throughout this work we often drop "elementary" and use the phrases EAU and (elementary) argumentative unit and argumentative component interchangeably.
}

our work investigates how (de-)contextualization of argumentative units affects automatic argumentative relation classification models.

Notions of context Various notions of context are being used in the area of argumentation mining. For example, Lippi and Torroni (2016) develop a context-independent claim detection system, where by context-independent they refer to a system which is not tailored to a specific topic (analogously, Levy et al. (2014) aim at contextdependency). Another notion of context concerns the graph context in which relations and EAUs are embedded (Kuribayashi et al., 2018). On the other hand, we adopt a more textual notion of context, that is we take a given EAU span as content and text which is not in the EAU span as context. This goes in the same direction as Stab and Gurevych (2014b, 2017); Persing and Ng (2016) and Aker et al. (2017) who incorporate features derived from EAU-surrounding text in their classification systems. However, they do not clearly separate between a word indicator feature extracted from within (or outside) the EAU span. For example, when computing features for an EAU, they also take into account EAU-preceding tokens. The preceding tokens, often contain shallow discourse markers which highlight the relationship between two EAUs (e.g., because, however, etc.).

To the best of our knowledge, prior work has not yet thoroughly investigated the impact of features extracted from the EAU vs. features extracted from the EAU-embedding context. Our work fills this gap and shows that the impact of contextual clues from the EAU context on classifier performance can be much greater than the impact of features extracted from the EAU content.

Context matters Nguyen and Litman (2016); Nguyen (2018) extract additional features from the text between source and target EAUs (on the StudentEssay-v01 data (Stab and Gurevych, 2014a)) which results in enhanced predictive performance. However, having seen the clear advantages of incorporating context (performancewise), we find that the downsides of incorporating context remain untold. In this work, we demonstrate that systems which are offered EAU context may be prone to neglect the EAU content, an issue that can have undesired effects.

Argumentative relation classification Argumentative relation classification (Mochales and 
Moens, 2011) is the task for which we aim to examine the context-content relationship. It is concerned with predicting and analyzing relations between argumentative units such as, for example, support or attack. Besides works discussed above (Nguyen and Litman, 2016; Stab and Gurevych, 2014b, 2017), this task has also been addressed by Cocarascu and Toni (2017) who develop a neural model to label the edge between two EAUs with $\{$ attack, support, $\varnothing\}$. The task has also been approached by taking global graph context into account. E.g., Hou and Jochim (2017) jointly model argument relation classification and stance classification in the DebatePedia ${ }^{3}$ corpus using Markov logic networks (Richardson and Domingos, 2006). Peldszus and Stede (2015) experiment with Microtexts and show that it can be beneficial to model argumentative relations jointly in a network with a minimum spanning tree decoding algorithm. Our work focuses on local relation prediction and labeling using the well-established StudentEssayv02 data (Stab and Gurevych, 2017) ${ }^{4}$ with 402 argumentative essays and thousands of annotated relations between EAUs.

\section{Argumentative Relation Prediction: Models and Features}

In this section, we describe different formulations of the argumentative relation classification task and describe features used by our replicated model. In order to test our hypotheses, we propose to group all features into three distinct types.

Three feature types: content-based; contentignorant; full access We categorize features of Stab and Gurevych (2017) into three types: (i) features derived from the context of the argumentative unit (e.g., leading and trailing tokens surrounding the EAU span), (ii) features derived from the argumentative unit's content (i.e., the EAU span), and (iii) a joint feature set consisting of the union of features from (i) and (ii). However, in (iii) we additionally include features that capture discourse structures that overlap the boundaries between an EAU and its surroundings.

Notations Henceforth we denote models that only make use of features of type (i), ignoring anything inside the EAU, as content-ignorant $(\mathcal{C I})$, and models that are given only features covering

\footnotetext{
${ }^{3}$ http: / / debatepedia.idebate.org/

${ }^{4}$ https: / / tinyurl.com/y269fq3k
}

the EAU span as content-based $(\mathcal{C B})$. A model that combines both is denoted by full-access $(\mathcal{F} \mathcal{A})$. We distinguish these different model types with a type-variable $\mathcal{T} \in\{\mathcal{C I}, \mathcal{C B}, \mathcal{F} \mathcal{A}\}$.

\subsection{Models}

Now, we introduce a classification of three different prediction models used in the argumentative relation prediction literature. We will inspect all of them and show that all can suffer from severe issues when focusing (too much) on the context.

The model $h$ adopts a discourse parsing view on argumentative relation prediction and predicts one outgoing edge for an argumentative unit (oneoutgoing edge). Model $f$ assumes a connected graph with argumentative units and is tasked with predicting edge labels for unit tuples (labeling relations in a graph). Finally, a model $g$ is given two (possibly) unrelated argumentative units and is tasked with predicting connections as well as edge labels (joint edge prediction and labeling).

One-outgoing edge Stab and Gurevych (2017) divide the task into relation prediction $l$ and relation class assignment $h$ :

$$
\begin{gathered}
l^{\mathcal{T}}: A \times A \rightarrow\{\text { linked }, \varnothing\} \\
h^{\mathcal{T}}: A \rightarrow\{\text { attack, support }\},
\end{gathered}
$$

which the authors describe as argumentative relation identification $(l)$ and stance detection $(h)$. In their experiments, $\mathcal{T}=F A$, i.e., no distinction is made between features that access only the argument content (EAU span) or only the EAU's embedding context, and some features also consider both (e.g., discourse features). This model adopts a parsing view on argumentative relation classification: every unit is allowed to have only one type of outgoing relation (this follows trivially from the fact that $h$ has only one input). Applying such a model to argumentative attack and support relations might impose unrealistic constraints on the resulting argumentation graph: A given premise might in fact attack or support several other premises. ${ }^{5}$ The approach may suffice for the case of student argumentative essays, where EAUs are well-framed in a discourse structure, but seems overly restrictive for many other scenarios.

\footnotetext{
${ }^{5}$ E.g., this decision will improve the living situation for children. It may also support elderly people with low income.
} 
Labeling relations in a graph Another way of framing the task, is to learn a function

$$
f^{\mathcal{T}}: A \times A \rightarrow\{\text { support, attack }\},
$$

Here, an argumentative unit is allowed to be in a attack or support relation to multiple other EAUs. Yet, both $h$ and $f$ assume that inputs are already linked and only the class of the link is unknown.

Joint edge prediction and labeling Thus, we might also model the task in a three-class classification setting to learn a more general function that performs relation prediction and classification jointly (see also, e.g., Lippi and Torroni (2016)):

$$
g^{\mathcal{T}}: A \times A \rightarrow\{\text { support, attack, } \varnothing\} .
$$

The model described by Eq. 4 is the most general one: not only does it assume a graph view on argumentative units and their relations (as does Eq. 3); in model formulation (Eq. 4), an argumentative unit can have no or multiple support or attack relations. It naturally allows for cases where an argumentative unit $a$ (supports $b \mid$ attacks $c \mid$ isunrelated-to $d$ ). Given a set of EAUs mined from different documents, this model enables us to construct a full-fledged argumentation graph.

\subsection{Feature implementation}

Our feature implementation follows the feature descriptions for Stance recognition and link identification in Stab and Gurevych (2017). These features and variations of them have been used successfully in several successive works (cf. Stab and Gurevych (2014b); Nguyen and Litman (2016); Aker et al. (2017)).

For any model the features are indexed by $I=$ $\{1, \ldots, N\}$. We create a function $\Phi: I \rightarrow \mathcal{T}$ which maps from feature indices to feature types. In other words, $\Phi$ tells us, for any given feature, whether it is content-based $(\mathcal{C B})$, content-ignorant $(\mathcal{C I})$ or full access $(\mathcal{F} \mathcal{A})$. The features for, e.g., the joint prediction model $g$ of type $\mathcal{C I}\left(g^{\mathcal{C I}}\right)$ can then simply be described as $\{i \in I \mid \Phi(i)=$ $\mathcal{C I}\}$. Recall that features computed on the basis of the EAU span are content-based $(\mathcal{C B})$, features from the EAU-surrounding text are contentignorant $(\mathcal{C I})$ and features computed from both are denoted by full-access $(\mathcal{F} \mathcal{A})$. Details on the extraction of features are provided below.

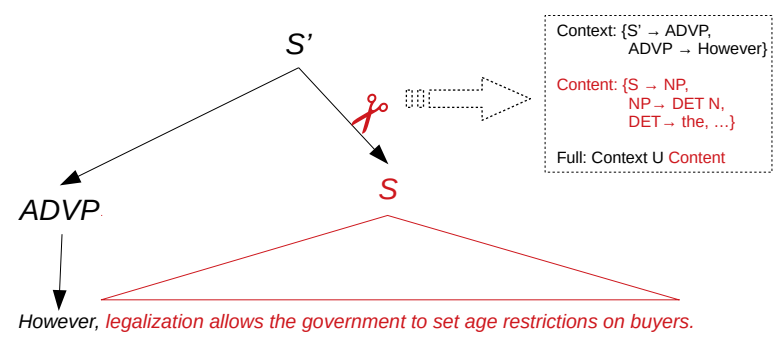

Figure 2: Production rule extraction from constituency parse for two different argumentative units.

Lexical features These consist of boolean values indicating whether a certain word appears in the argumentative source or target EAU or both (and separately, their contexts). More precisely, for any classification instance we extract uni-grams from within the span of the EAU (if $\mathcal{T}=\mathcal{C B}$ ) or solely from the sentence-context surrounding the EAUs (if $\mathcal{T}=\mathcal{C} \mathcal{I}$ ). Words which occur in both bags are only visible in the full-access setup $\mathcal{T}=\mathcal{F} \mathcal{A}$ and are modeled as binary indicators.

Syntactic features Such features consist of syntactic production rules extracted from constituency trees - they are modelled analogously to the lexical features as a bag of production rules. To make a clear division between features derived from the EAU embedding context and features derived from within the EAU span, we divide the constituency tree in two parts, as is illustrated in Figure 2. If the EAU is embedded in a covering sentence, we cut the syntax tree at the corresponding edge ( $x_{0}$ in Figure 2 ). In this example, the content-ignorant $(\mathcal{C I})$ bag-of-word production rule representation includes the rules $S \rightarrow A D V P$ and $A D V P \rightarrow$ however. Analogously to the lexical features, the production rules are modeled as binary indicator features. ${ }^{6}$

Structural These features describe shallow statistics such as the ratio of argumentative unit tokens compared to sentence tokens or the position of the argumentative unit in the paragraph. We set these features to zero for the content representation of the argumentative unit and replicate those features that allow us to treat the argumen-

\footnotetext{
${ }^{6} \mathrm{~A}$ notable insight from our experiments is that the production rule features have a considerable intersection with lexical features. This is due to the terminal production rules, which correspond to the leaves of the constituency tree. This explains the surprisingly high scores for production rule features in the production-rule-only ablation experiments in e.g., Stab and Gurevych (2014b, 2017).
} 
tative unit as a black-box. For example, in the content-based $(\mathcal{C B})$ system that has access only to the EAU, we can compute the \#tokens in the EAU, but not the \#tokens in EAU divided by \#tokens in the sentence. The latter feature is only accessible in the full access system variants. Hence, in the content-based $(\mathcal{C B})$ system most of these statistics are set to zero since they cannot be computed by considering only the EAU span.

Discourse For the content-based representation we retrieve only discourse relations that are confined within the span of the argumentative unit. In the very frequent case that discourse features cross the boundaries of embedding context and EAU span, we only take them into account for $\mathcal{F} \mathcal{A}$.

Embeddings We use the element-wise sum of 300-dimensional pre-trained GloVe vectors (Pennington et al., 2014) corresponding to the words within the EAU span $(\mathcal{C B})$ and the words of the EAU-surrounding context $(\mathcal{C I})$. Additionally, we compute the element-wise subtraction of the source EAU vector from the target EAU vector, with the aim of modelling directions in distributional space, similarly to Mikolov et al. (2013). Words with no corresponding pre-trained word vector and empty sequences (e.g., no preceding context available) are treated as a zero-vector.

Sentiment Tree-based sentiment annotations are sentiment scores assigned to nodes in constituency parse trees (Socher et al., 2013). We represent these scores by a one-hot vector of dimension 5 ( 5 is very positive, 1 is very negative). We determine the contextual $(\mathcal{C I})$ sentiment by looking at the highest possible node of the context which does not contain the EAU (ADVP in Figure 2). The sentiment for an EAU span $(\mathcal{C B})$ is assigned to the highest possible node covering the EAU span which does not contain the context subtree ( $\mathrm{S}$ in Figure 2). The full-access $(\mathcal{F} \mathcal{A})$ score is assigned to the lowest possible node which covers both the EAU span and its surrounding context ( $S$ ' in Figure 2). Next to the sentiment scores for the selected tree nodes and analogously to the word embeddings, we also calculate the elementwise subtraction of the one-hot sentiment source vectors from the one-hot sentiment target vectors. This results in three additional vectors corresponding to $\mathcal{C B}, \mathcal{C I}$ and $\mathcal{F A}$ difference vectors.

\begin{tabular}{l|rr|rr} 
& \multicolumn{2}{|c}{ \#train } & \multicolumn{2}{c}{ \#test } \\
\hline model & $h \& f$ & $g$ & $h \& f$ & $g$ \\
\hline documents & 322 & 322 & 80 & 80 \\
support & 3820 & 3820 & 1021 & 1021 \\
attack & 405 & 405 & 92 & 92 \\
$\varnothing$ & - & 5474 & - & 1622
\end{tabular}

Table 1: Data set statistics.

\section{Experiments}

Data and pre-processing We use the corpus of 402 persuasive essays which were annotated with argumentative units, their stances towards the topic and argumentative relations (Stab and Gurevych, 2017). The data is suited for our experiments because the annotators were explicitly asked to provide annotations on a clausal level. This entails that contextual clues tend not to be contained in the annotated span (e.g., only people should not smoke is annotated as EAU in the sentence Therefore, people should not smoke.). In this work, we are concerned with classifying relations between argumentative units into support or attack and thus do not consider other annotations. For feature extraction, we process all documents with Stanford CoreNLP (Manning et al., 2014) with the following annotation layers: sentence tokenize, word tokenize, constituency parse and constituency-sentiment. For extraction of the discourse-features, we proceed by parsing all documents with the PDTB-parser ${ }^{7}$ developed by Lin et al. (2014). For the joint task of predicting three link classes (including a non-linked class), we extract as non-linked EAU pairs all EAU pairs which are not linked on a document level. Data set statistics are displayed in Table 1.

Setup As explained in $\S 3$, we are interested in three distinct configurations of the argumentative relation classifier: content-based $(\mathcal{C B})$, contentignorant $(\mathcal{C} \mathcal{I})$ and full-access $(\mathcal{F} \mathcal{A})$. Naturally, we would expect the latter to perform best and perhaps we would also expect $\mathcal{C B}$ to outperform $\mathcal{C I}$ - a system which has no access to the argumentative unit internals whatsoever should not be able to confidently determine relations between them.Note that some features are only available to $\mathcal{F} \mathcal{A}$, which is the case when features cross con-

\footnotetext{
${ }^{7}$ https://github.com/WING-NUS / pdtb-parser
} 


\begin{tabular}{lrrr}
\hline system & $\mathrm{F}_{\text {sup }}$ & $\mathrm{F} 1_{\text {att }}$ & macro F1 \\
\hline S\&G16 & 94.7 & 41.3 & 68.0 \\
replicated $\left(h^{\mathcal{F} \mathcal{A})}\right.$ & 94.7 & 44.0 & 69.3 \\
\hline
\end{tabular}

Table 2: Baseline system replication results.

\begin{tabular}{llllll}
\hline model & $\mathcal{T}$ & $\mathrm{F}_{\text {sup }}$ & $\mathrm{F1}_{\text {att }}$ & $\mathrm{F} 1_{\varnothing}$ & macro F1 \\
\hline$h$ & $\mathrm{mfs}$ & 95.7 & 0 & - & 47.8 \\
& $\mathcal{C B}$ & 92.9 & 21.7 & - & $57.3^{\dagger}$ \\
& $\mathcal{C} \mathcal{I}$ & 95.0 & 38.6 & - & $67.0^{\dagger \ddagger}$ \\
& $\mathcal{F} \mathcal{A}$ & 94.7 & 44.0 & - & $69.3^{\dagger \ddagger}$ \\
\hline$f$ & $\mathrm{mfs}$ & 95.7 & 0 & - & 47.8 \\
& $\mathcal{C B}$ & 92.3 & 20.3 & - & $56.3^{\dagger}$ \\
& $\mathcal{C} \mathcal{I}$ & 96.1 & 41.7 & - & $70.8^{\dagger \ddagger}$ \\
& $\mathcal{F} \mathcal{A}$ & 94.4 & 42.4 & - & $68.5^{\dagger \ddagger}$ \\
\hline$g$ & $\mathrm{mfs}$ & 0 & 0 & 74.5 & 8.3 \\
& $\mathcal{C B}$ & 54.3 & 9.9 & 65.0 & $43.4^{\dagger}$ \\
& $\mathcal{C} \mathcal{I}$ & 63.0 & 34.8 & 76.5 & $59.3^{\dagger \ddagger}$ \\
& $\mathcal{F} \mathcal{A}$ & 46.6 & 32.3 & 73.1 & $56.1^{\dagger \ddagger}$ \\
\hline
\end{tabular}

Table 3: Argumentative relation classification models $h, f, g$ with different access to content and context; models of type $\mathcal{C I}$ (content-ignorant) have no access to the EAU span. $\dagger$ : significantly better than $\mathrm{mfs}$ baseline $(p<0.005)$; $\ddagger$ significantly better than content-based $(p<0.005)$.

text and argumentative unit spans (e.g., some of the discourse features), thereby resisting a clear categorization into $\mathcal{C B}$ or $\mathcal{C I}$. Same as most prior work, we use an SVM to learn the feature weights.

\subsection{Results}

Replication experiments Our first step towards our main experiments is to replicate the competitive argumentative relation classifier of Stab and Gurevych $(2017,2014 b)$. Hence, for comparison purposes, we first formulate the task exactly as it was done in this prior work, using the model formulation in Eq. 2, which determines the type of outgoing edge from a source (i.e., tree-like view).

The results in Table 2 confirm the results of Stab and Gurevych (2017) and suggest that we successfully replicated a large proportion of their features.

Main results The results for all three prediction settings (one outgoing edge: $h$, support/attack: $f$ and support/attack/neither: $g$ ) across all type variables $(\mathcal{C B}, \mathcal{C I}$ and $\mathcal{F} \mathcal{A})$ are displayed in Table 3. All models significantly outperform the majority baseline with respect to macro F1. Intriguingly, the content-ignorant models $(\mathcal{C I})$ always perform significantly better than the models which only have access to the EAUs' content $(\mathcal{C B}, p<0.005)$. In the most general task formulation $(g)$, we observe that $\mathcal{C I}$ even significantly outperforms the model which has maximum access (seeing both EAU spans and surrounding contexts: $\mathcal{F} \mathcal{A}$ ).

At first glance, the results of the purely EAU focused systems $(\mathcal{C B})$ are disappointing, since they fall far behind their competitors. On the other hand, their F1 scores are not devastatingly bad. The strong most-frequent-class baseline is significantly outperformed by the content-based $(\mathcal{C B})$ system, across all three prediction settings.

In summary our findings are as follows: (i) models which see the EAU span (content-based, $\mathcal{C B}$ ) are significantly outperformed by models that have no access to the span itself (content-ignorant, $\mathcal{C I}$ ) across all settings; (ii) in two of three prediction settings ( $f$ and $g$ ), the model which only has access to the context even outperforms the model that has access to all information in the input. The fact that using features derived exclusively from the EAU embedding context $(\mathcal{C I})$ can lead to better results than using a full feature-system $(\mathcal{F} \mathcal{A})$ suggests that some information from the EAU can even be harmful. Why this is the case, we cannot answer exactly. A plausible cause might be related to the smaller dimension of the feature space, which makes the SVM less likely to overfit. Still, this finding comes as a surprise and calls for further investigation in future work.

Robustness tests A system for argumentative relation classification can be applied in one of two settings: single-document or cross-document, as illustrated in Figure 3: in the first case (top), a system is tasked to classify EAUs that appear linearly in one document - here contextual clues can often highlight the relationship between two units. This is the setting we have been considering up to now. However, in the second scenario (bottom), we have moved away from the closed singledocument setting and ask the system to classify two EAUs extracted from different document contexts. This setting applies, for instance, when we are mining arguments from multiple sources.

In both cases, however, a system that relies more on contextual clues than on the content expressed in the EAUs is problematic: in the singledocument setting, such a system will rely on discourse indicators - whether or not they are justi- 


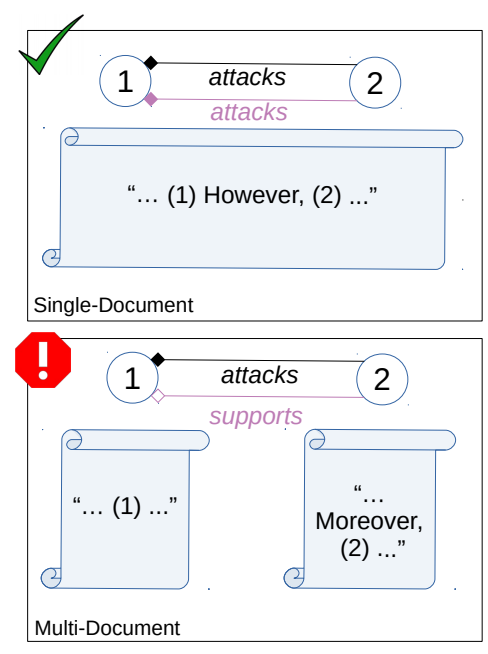

Figure 3: Single-document (top) vs. cross-document (bottom) argumentative relation classification. Black edge: gold label; purple edge: predicted label.

fied by content - and can thus easily be fooled.

In the cross-document setting, discourse-based indicators - being inherently defined with respect to their internal document context - do not have a defined rhetorical function with respect to EAUs in a separate document and thus a system that has learned to rely on such markers within a singledocument setting can be seriously misled. We believe that the cross-document setting should be an important goal in argumentation analysis, since it generalizes better to many debates of interest, where EAUs can be found scattered across thousands of documents. For example, for the topic of legalizing marijuana, EAUs may be mined from millions of documents and thus their relations may naturally extend across document boundaries. If a system learns to over-proportionally attend to the EAUs' surrounding contexts it is prone to making many errors. ${ }^{8}$

In what follows we are simulating the effects that an overly context-sensitive classifier could have in a cross-document setting, by modifying our experimental setting, and study the effects on the different model types: In one setup - we call it randomized-context - we systematically distort the context of our testing instances by exchanging the context in a randomized manner; in the other setting - called no-context, we are deleting the context around the ADUs to be classified.

\footnotetext{
${ }^{8}$ In fact, similar considerations also apply when moving from argumentative monologue to dialogue, i.e., in interactive debates. Again, systems need to be able to detect relations between EAUs uttered by different speakers and independently from the speaker-specific utterance context.
}

Randomized-context simulates an open world debate where argumentative units may occur in different contexts, sometimes with discourse markers indicating an opposite class. In other words, in this setting we want to examine effects when porting a context-sensitive system to a multi-document setting. ${ }^{9}$ For example, as seen in Figure 3, the context of an argumentative unit may change from "However" to "Moreover" - which can happen naturally in open debates. The results are displayed in Figure 4 . In the standard setting (Figure 4a), the models that have access to the context besides the content $(\mathcal{F} \mathcal{A})$ and the models that are only allowed to access the context $(\mathcal{C I})$, always perform better than the content-based models $(\mathcal{C B})$ (bars above zero). However, when we randomly flip contexts of the test instances (Figure 4b), or suppress them entirely (Figure 4c), the opposite picture emerges: the content-based models always outperform the other models. For some classes (support, $\varnothing$ ) the difference can exceed $50 \mathrm{~F} 1$ percentage points. These two studies, where testing examples are varied regarding their context (randomized-context or no-context) simulates what can be expected if we apply our systems for relation class assignment to EAUs stemming from heterogeneous sources. While the performances of a purely content-based model naturally stays stable, the performance of the other systems decrease notably - they perform worse than the content-based model.

Feature investigation We calculate the ANOVA classification F scores of the features with respect to our three task formulations $h, g$ and $f$. The $F$ percentiles of features extracted from the EAU surrounding text $(\mathcal{C I})$ and features extracted from the EAU span $(\mathcal{C B})$, are displayed in Figure 5.

It clearly stands out that features obtained from the EAU surrounding context $(\mathcal{C I})$ are assigned much higher scores compared to features stemming from the EAU span $(\mathcal{C B})$. This holds true for all three task formulations and provides further evidence that models - when given the option - put a strong focus on contextual clues while neglecting the information provided by the EAU span itself.

\section{Discussion}

While competitive systems for argumentative relation classification are considered to be robust, our

\footnotetext{
${ }^{9}$ We concede that this is an artificial setup, but defer more realistic cross-document experiments to future work.
} 


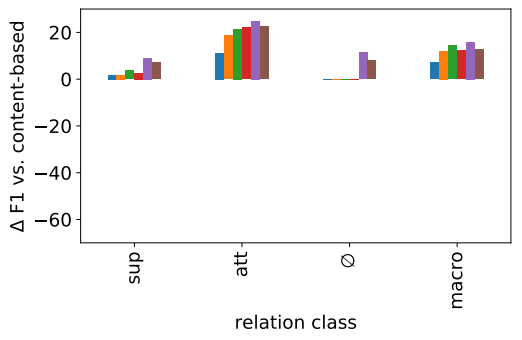

(a) Standard setting.

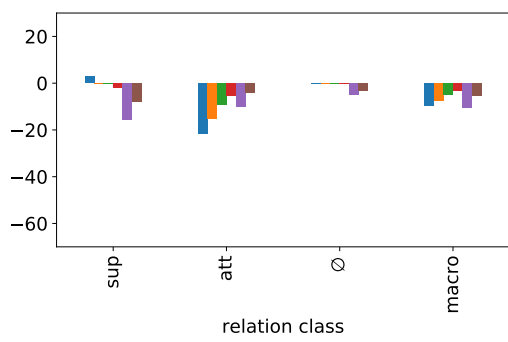

(b) Randomized-context test.

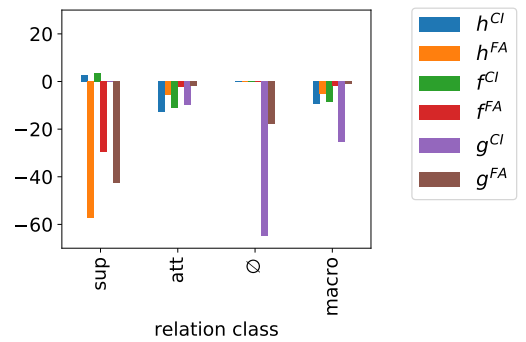

(c) No-context test.

Figure 4: Randomized-context test set: models are applied to testing instances with randomly flipped contexts. No-context test set: models can only access the EAU span of a testing instance. A bar below/above zero means that a system that can access context (content-ignorant $\mathcal{C I}$ or full-access $\mathcal{F} \mathcal{A}$ ) is worse/better than the content-based baseline $\mathcal{C B}$ that only has access to the EAU span (its performance is not affected by modified context, cf. Tab. 3 ).

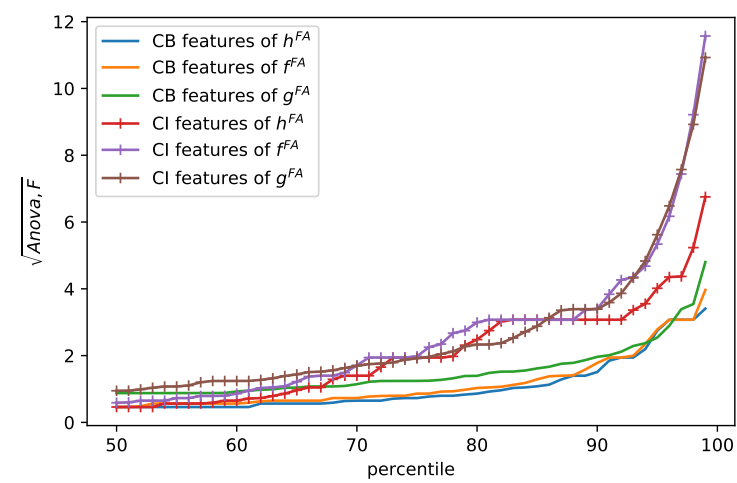

Figure 5: ANOVA F score percentiles for contentbased vs. content-ignorant features in the training data. A higher feature score suggests greater predictive capacity.

experiments have shown that despite confidenceinspiring scores on unseen testing data, such systems can easily be fooled - they can deliver strong performance scores although the classifier does not have access to the content of the EAUs. In this respect, we have provided evidence that there is a danger in case models focus too much on rhetorical indicators, in detriment of the context. Thus, the following question arises: How can we prevent argumentation models from modeling arguments or argumentative units and their relations in overly naïve ways? A simple and intuitive way is to dissect EAUs from their surrounding document context. Models trained on data that is restricted to the EAUs' content will be forced to focus on the content of EAUs. We believe that this will enhance the robustness of such models and allows them to generalize to cross-document argument relation classification. The corpus of student essays makes such transformations straightforward: only the
EAUs were annotated (e.g., "However, $[\arg \mathrm{A}]$ "). If annotations extend over the EAUs (e.g., only

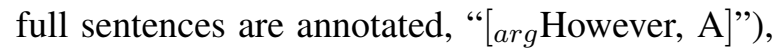
such transformations could be performed automatically after a discourse parsing step. When inspecting the student essays corpus, we further observed that an EAU mining step should involve coreference resolution to better capture relations between EAUs that involve anaphors (e.g., "Exercising makes you feel better" and "It [Exercising] increases endorphine levels”).

Thus, in order to conduct real-world end-toend argumentation relation mining for a given topic, we envision a system that addresses three steps: (i) mining of EAUs and (ii) replacement of pronouns in EAUs with referenced entities (e.g., It is healthy $\rightarrow$ Excercise is healthy). Finally (iii), given the cross product of mined EAUs we can apply a model of type $g$ to construct a fullfledged argumentation graph, possibly spanning multiple documents. ${ }^{10}$ We have shown that in order to properly perform step (iii), we need stronger models that are able to better model EAU contents. Hence, we encourage the argumentation community to test their systems on a decontextualized version of the student essays, including the proposed - and possibly further extended - testing setups, to challenge the semantic representation and reasoning capacities of argument analysis models. This will lead to more realistic performance estimates and increased robustness of systems when addressing desirable multi-document tasks.

\footnotetext{
${ }^{10} \mathrm{cf}$. Peldszus and Stede (2015) for graph prediction within single documents.
} 


\section{Conclusion}

We have shown that systems which put too much focus on discourse information may be easily fooled - an issue which has severe implications when systems are applied to cross-document argumentative relation classification tasks. The strong reliance on contextual clues is also problematic in single-document contexts, where systems can run a risk of assigning relation labels relying on contextual and rhetorical effects - instead of focusing on content. Hence, we propose that researchers test their argumentative relation classification systems on two alternative versions of the StudentEssay data that reflect different access levels. (i) EAU-span only, where systems only see the EAU spans and (ii) context-only, where systems can only see the EAU-surrounding context. These complementary settings will (i) challenge the semantic capacities of a system, and (ii) unveil the extent to which a system is focusing on the discourse context when making decisions. We will offer our testing environments to the research community through a platform that provides datasets and scripts and a table to trace the results of content-based systems. ${ }^{11}$

\section{Acknowledgments}

This work has been supported by the German Research Foundation as part of the Research Training Group Adaptive Preparation of Information from Heterogeneous Sources (AIPHES) under grant no. GRK 1994/1 and by the Leibniz ScienceCampus "Empirical Linguistics and Computational Language Modeling", supported by the Leibniz Association under grant no. SAS-2015-IDS-LWC and by the Ministry of Science, Research, and Art of Baden-Württemberg.

\section{References}

Ahmet Aker, Alfred Sliwa, Yuan Ma, Ruishen Lui, Niravkumar Borad, Seyedeh Ziyaei, and Mina Ghobadi. 2017. What works and what does not: Classifier and feature analysis for argument mining. In Proceedings of the 4th Workshop on Argument Mining, pages 91-96, Copenhagen, Denmark.

M. Azar. 1999. Argumentative text as rhetorical structure: An application of rhetorical structure theory. Argumentation, 13(1):97-114.

\footnotetext{
${ }^{11}$ http://explain.cl.uni-heidelberg.de/
}

Oana Cocarascu and Francesca Toni. 2017. Identifying attack and support argumentative relations using deep learning. In Proceedings of the 2017 Conference on Empirical Methods in Natural Language Processing, pages 1374-1379.

Nancy Green, Kevin Ashley, Diane Litman, Chris Reed, and Vern Walker, editors. 2014. Proceedings of the First Workshop on Argumentation Mining. Association for Computational Linguistics, Baltimore, Maryland.

Nancy L Green. 2010. Representation of argumentation in text with rhetorical structure theory. Argumentation, 24(2):181-196.

Yufang Hou and Charles Jochim. 2017. Argument relation classification using a joint inference model. In Proceedings of the 4th Workshop on Argument Mining, pages 60-66.

Tatsuki Kuribayashi, Paul Reisert, Naoya Inoue, and Kentaro Inui. 2018. Towards exploiting argumentative context for argumentative relation identification. In Proceedings of the Annual Meeting of the Association for Natural Language Processing NLP, pages 284-287.

Ran Levy, Yonatan Bilu, Daniel Hershcovich, Ehud Aharoni, and Noam Slonim. 2014. Context dependent claim detection. In Proceedings of COLING 2014, the 25th International Conference on Computational Linguistics: Technical Papers, pages 14891500, Dublin, Ireland. Dublin City University and Association for Computational Linguistics.

Ziheng Lin, Hwee Tou Ng, and Min-Yen Kan. 2014. A pdtb-styled end-to-end discourse parser. Natural Language Engineering, 20(2):151-184.

Marco Lippi and Paolo Torroni. 2016. Argumentation mining: State of the art and emerging trends. ACM Transactions on Internet Technology (TOIT), 16(2):10.

William C Mann and Sandra A Thompson. 1987. Rhetorical structure theory: Description and construction of text structures. In Natural language generation, pages 85-95. Springer.

Christopher Manning, Mihai Surdeanu, John Bauer, Jenny Finkel, Steven Bethard, and David McClosky. 2014. The Stanford CoreNLP natural language processing toolkit. In Proceedings of 52nd Annual Meeting of the Association for Computational Linguistics: System Demonstrations, pages 55-60, Baltimore, Maryland.

Tomas Mikolov, Ilya Sutskever, Kai Chen, Greg S Corrado, and Jeff Dean. 2013. Distributed representations of words and phrases and their compositionality. In Advances in neural information processing systems, pages 3111-3119.

Raquel Mochales and Marie-Francine Moens. 2011. Argumentation mining. Artificial Intelligence and Law, 19(1):1-22. 
Huy Nguyen. 2018. Context-aware Argument Mining and Its Applications in Education. Ph.D. thesis, University of Pittsburgh.

Huy Nguyen and Diane Litman. 2016. Context-aware argumentative relation mining. In Proceedings of the 54th Annual Meeting of the Association for Computational Linguistics (Volume 1: Long Papers), volume 1, pages 1127-1137.

Andreas Peldszus and Manfred Stede. 2013. From argument diagrams to argumentation mining in texts: A survey. International Journal of Cognitive Informatics and Natural Intelligence (IJCINI), 7(1):1-31.

Andreas Peldszus and Manfred Stede. 2015. Joint prediction in mst-style discourse parsing for argumentation mining. In Proceedings of the 2015 Conference on Empirical Methods in Natural Language Processing, pages 938-948.

Andreas Peldszus and Manfred Stede. 2016. An annotated corpus of argumentative microtexts. In D. Mohammed and M. Lewinski, editors, Argumentation and Reasoned Action - Proc. of the 1st European Conference on Argumentation, Lisbon, 2015. College Publications, London.

Jeffrey Pennington, Richard Socher, and Christopher Manning. 2014. Glove: Global vectors for word representation. In Proceedings of the 2014 conference on Empirical Methods in Natural Language Processing (EMNLP), pages 1532-1543.

Isaac Persing and Vincent Ng. 2016. End-to-end argumentation mining in student essays. In Proceedings of the 2016 Conference of the North American Chapter of the Association for Computational Linguistics: Human Language Technologies, pages 1384-1394, San Diego, California. Association for Computational Linguistics.

Mohammad Sadegh Rasooli and Joel R. Tetreault. 2015. Yara parser: A fast and accurate dependency parser. Computing Research Repository, arXiv:1503.06733. Version 2.

Matthew Richardson and Pedro Domingos. 2006. Markov logic networks. Machine learning, 62(12):107-136.

Richard Socher, Alex Perelygin, Jean Wu, Jason Chuang, Christopher D. Manning, Andrew Ng, and Christopher Potts. 2013. Recursive deep models for semantic compositionality over a sentiment treebank. In Proceedings of the 2013 Conference on Empirical Methods in Natural Language Processing, pages 1631-1642, Seattle, Washington, USA.

Christian Stab and Iryna Gurevych. 2014a. Annotating argument components and relations in persuasive essays. In Proceedings of COLING 2014, the 25th International Conference on Computational Linguistics: Technical Papers, pages 1501-1510, Dublin, Ireland. Dublin City University and Association for Computational Linguistics.
Christian Stab and Iryna Gurevych. 2014b. Identifying argumentative discourse structures in persuasive essays. In Proceedings of the 2014 Conference on Empirical Methods in Natural Language Processing (EMNLP), pages 46-56.

Christian Stab and Iryna Gurevych. 2017. Parsing argumentation structures in persuasive essays. Computational Linguistics, 43(3):619-659.

Christian Stab, Tristan Miller, Benjamin Schiller, Pranav Rai, and Iryna Gurevych. 2018. Cross-topic argument mining from heterogeneous sources. In Proceedings of the 2018 Conference on Empirical Methods in Natural Language Processing, pages 3664-3674.

Henning Wachsmuth, Manfred Stede, Roxanne El Baff, Khalid Al Khatib, Maria Skeppstedt, and Benno Stein. 2018. Argumentation synthesis following rhetorical strategies. In Proceedings of the 27th International Conference on Computational Linguistics, pages 3753-3765. 